\title{
Analysis of Homogeneous Waveguides via the Meshless Radial Basis Function Generated-Finite Difference Method
}

\author{
José A. Pereda and Ana Grande
}

\begin{abstract}
The radial basis function generated-finite difference (RBF-FD) method is applied to the analysis of homogenous waveguides. To this end, the Helmholtz equation and the boundary conditions are collocated on the waveguide cross section. At each collocation node, derivatives are locally approximated by RBF-FD formulas based on polyharmonic splines supplemented with high-degree polynomials. As a result, a sparse matrix eigenvalue problem is obtained which allows cutoff wavenumbers and axial fields to be calculated. To illustrate the accuracy of the method, we consider a semicircular and an eccentric circular waveguides.
\end{abstract}

Index Terms-Meshless methods, radial basis function generated-finite difference method, homogeneous waveguides, cutoff wavenumbers

\section{INTRODUCTION}

The numerical solution of partial differential equations by classical finite-difference (FD) methods is based on using a mesh to approximate unknowns and their derivatives. Alternatively, in meshless methods the approximation is based on scattered nodes without any mesh connectivity, which provides more flexibility and simplicity in complex geometries [1].

During the last decades, meshless methods have been successfully applied in many branches of science and engineering. However they still remain relatively less developed within the Computational Electromagnetics field [2]-[4].

Among the meshless methods, the radial basis function generated-finite difference (RBF-FD) method can be viewed as a generalization of the classical FD method from structured meshes to scattered node sets. It has been recently shown that the RBF-FD method allows high-order local FD formulas to be obtained by using interpolants based on polyharmonic spline (PHS) RBFs supplemented with high-degree polynomials [5], [6].

In this letter, the RBF-FD method is applied to the analysis of homogenous waveguides. To this end, the collocation approach is applied to the Helmholtz equation with appropriate boundary conditions (BCs). At each collocation node, derivatives are locally approximated by suitable RBF-FD

Manuscript received September 18, 2019; revised November 19, 2019 accepted December 6, 2019. This work was supported in part by the Spanish Government (MCIU/AEI) and the European Commission (FEDER, UE) under Research Projects PGC2018-098350-B-C21 and PGC2018-098350-B-C22.

J. A. Pereda is with the Dpto. de Ingeniería de Comunicaciones (DICOM), Universidad de Cantabria, Avda. Los Castros s/n, 39005 Santander, Cantabria, Spain (email: peredaj@unican.es).

A. Grande is with the Dpto. de Electricidad y Electrónica, Universidad de Valladolid, 47011 Valladolid, Spain (email: agrande@uva.es).

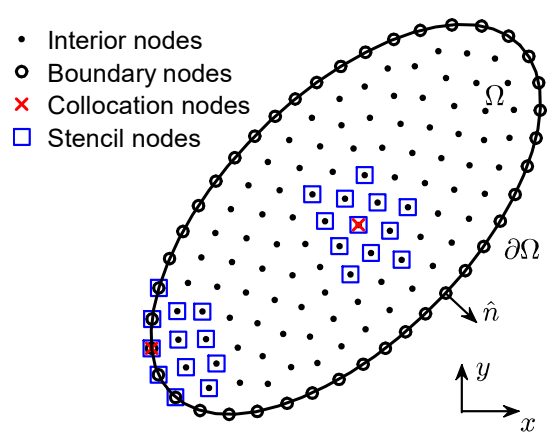

Fig. 1. Two different 12-node RBF-FD stencils in a waveguide with cross section $\Omega$ and boundary $\partial \Omega$. One stencil belongs to a collocation node in the domain $\Omega$ and the other one to a collocation node on the boundary $\partial \Omega$.

expressions. As a result, a sparse matrix eigenvalue problem is obtained which allows cutoff wavenumbers and axial fields to be calculated. To illustrate the accuracy of the method, we consider a semicircular and an eccentric circular waveguides.

Other meshless methods based on RBFs have recently been applied to the analysis of homogeneous waveguides (see [7] and references within). Comparing to them, the RBF-FD method adopted here does not require optimal shape parameter calculation. Additionally, polynomials up to a high degree are appended, which largely control the rate of convergence of the solution.

\section{THEORY}

Electromagnetic waves in a homogeneous waveguide with cross section $\Omega$ and perfect electric conductor boundary $\partial \Omega$ are determined by solving the Helmholtz eigenvalue problem:

$$
\begin{aligned}
L u(\vec{r}) & =-k_{c}^{2} u(\vec{r}), \quad \vec{r} \in \Omega \\
L_{B} u(\vec{r}) & =0, \quad \vec{r} \in \partial \Omega
\end{aligned}
$$

where $L$ is the Laplacian in the transverse $(x-y)$ plane, $k_{c}$ is the cutoff wavenumber and $u=E_{z}, H_{z}$ for TM and TE modes, respectively. The boundary operator $L_{B}$ is related to the type of mode. TM modes are subjected to Dirichlet BCs, $E_{z}=0$, while TE modes to Neumann BCs, $\nabla H_{z} \cdot \hat{n}=0$.

With the goal of approximating (1) by a matrix eigenvalue problem, (1a) is collocated at $N_{I}$ distinct node points in $\Omega$, and (1b) at $N_{B}$ distinct node points on $\partial \Omega$, with $N_{I}+N_{B}=N$. As shown in Fig. 1, at each collocation node $\vec{r}_{c}, L u\left(\right.$ or $L_{B} u$ ) is 
approximated by an FD formula with a stencil formed by the $N_{s}$ closest node points, including the collocation node itself:

$$
\left.L u(\vec{r})\right|_{r_{c}} \simeq \sum_{i=1}^{N_{s}} w_{i} u_{i}=\boldsymbol{w} \boldsymbol{u}_{s}
$$

where $u_{i} \equiv u\left(\vec{r}_{i}\right)$ are the values of $u$ at the stencil points, $w_{i}$ are the weights and $N_{s}$ is the stencil size $\left(N_{s} \ll N\right)$. In (2), $\boldsymbol{w}=\left[w_{1}, \ldots, w_{N s}\right]$ and $\boldsymbol{u}_{s}=\left[u_{1}, \ldots, u_{N_{s}}\right]^{T}$.

The calculation of $\boldsymbol{w}$ begins by considering an interpolant of the form [5], [6]

$$
u(\vec{r})=\sum_{j=1}^{N_{s}} a_{j} \phi_{j}\left(\left\|\vec{r}-\vec{r}_{j}\right\|\right)+\sum_{j=1}^{N_{p}} b_{j} p_{j}(\vec{r})
$$

where $a_{j}$ and $b_{j}$ are coefficients. The functions $p_{j}(\vec{r})$ are all the $N_{p}=\left(\begin{array}{c}d+2 \\ d\end{array}\right)$ bivariate monomials up to degree $d$. For instance, for $d=3$ there are $N_{p}=10$ monomials: $1, x, y, x y, x^{2}, y^{2}, x^{2} y, x y^{2}, x^{3}$ and $y^{3}$. The RBFs $\phi_{j}\left(\left\|\vec{r}-\vec{r}_{j}\right\|\right)$ are $N_{s}$ PHS centered at each node of the stencil $\vec{r}_{j}$, and $\|\cdot\|$ denotes the Euclidean norm. The PHS radial functions are defined as $\phi(r)=r^{m}$ if $m$ is odd, and $\phi(r)=$ $r^{m} \ln r$ if $m$ is even. For simplicity, only the odd case is considered here.

To obtain $a_{j}$ and $b_{j}$, the interpolant (3) is enforced to be exact at the $N_{s}$ nodes of the stencil with the additional constrains for the polynomials:

$$
\sum_{j=1}^{N_{s}} a_{j} p_{i}\left(\vec{r}_{j}\right)=0 \quad\left(i=1, \ldots, N_{p}\right) .
$$

This results in the following block linear system

$$
\left[\begin{array}{cc}
\Phi & P \\
P^{T} & 0
\end{array}\right]\left[\begin{array}{l}
\boldsymbol{a} \\
\boldsymbol{b}
\end{array}\right]=\left[\begin{array}{c}
\boldsymbol{u}_{s} \\
\mathbf{0}
\end{array}\right]
$$

where $\Phi$ is a symmetric $N_{s} \times N_{s}$ RBF interpolation matrix with elements $\Phi_{i j}=\phi_{j}\left(\left\|\vec{r}_{i}-\vec{r}_{j}\right\|\right)=\left\|\vec{r}_{i}-\vec{r}_{j}\right\|^{m}, P$ is a $N_{s} \times N_{p}$ matrix with $P_{i j}=p_{j}\left(\vec{r}_{i}\right)$ and, $\boldsymbol{a}$ and $\boldsymbol{b}$ are vectors given by $\boldsymbol{a}=\left[a_{1}, \ldots, a_{N_{s}}\right]^{T}$ and $\boldsymbol{b}=\left[b_{1}, \ldots, b_{N_{p}}\right]^{T}$.

Applying the differential operator $L$ to the interpolant in (3) and evaluating at $\vec{r}_{c}$, we get

$$
\begin{aligned}
\left.L u(\vec{r})\right|_{\vec{r}_{c}} & =\left.\sum_{j=1}^{N_{s}} a_{j} L \phi_{j}\left(\left\|\vec{r}-\vec{r}_{j}\right\|\right)\right|_{\vec{r}_{c}}+\left.\sum_{j=1}^{N_{p}} b_{j} L p_{j}(\vec{r})\right|_{\vec{r}_{c}} \\
& =\left[\begin{array}{ll}
\boldsymbol{l}_{\phi} & \boldsymbol{l}_{p}
\end{array}\right]\left[\begin{array}{c}
\boldsymbol{a} \\
\boldsymbol{b}
\end{array}\right]
\end{aligned}
$$

where

$$
\begin{aligned}
\boldsymbol{l}_{\phi} & =\left[\left.L \phi_{1}\left(\left\|\vec{r}-\vec{r}_{1}\right\|\right)\right|_{\vec{r}_{c}}, \ldots,\left.L \phi_{N_{s}}\left(\left\|\vec{r}-\vec{r}_{N_{s}}\right\|\right)\right|_{\vec{r}_{c}}\right], \\
\boldsymbol{l}_{p} & =\left[\left.L p_{1}(\vec{r})\right|_{\vec{r}_{c}}, \ldots,\left.L p_{N_{p}}(\vec{r})\right|_{\vec{r}_{c}}\right] .
\end{aligned}
$$

Solving (5) for $\left[\begin{array}{ll}a & b\end{array}\right]^{T}$, substituting the result into (6) and comparing with (2), the following linear system is obtained

$$
\left[\begin{array}{cc}
\Phi & P \\
P^{T} & 0
\end{array}\right]\left[\begin{array}{l}
\boldsymbol{w}^{T} \\
\boldsymbol{v}^{T}
\end{array}\right]=\left[\begin{array}{c}
\boldsymbol{l}_{\phi}^{T} \\
\boldsymbol{l}_{p}^{T}
\end{array}\right]
$$

where $\boldsymbol{w}$ contains the sought weights and the coefficients in $v$ are discarded after solving.

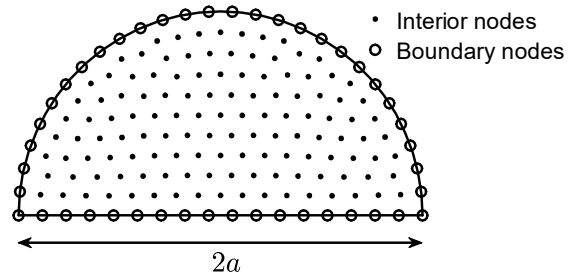

Fig. 2. Cross section of a semicircular waveguide of radius $a$.

By solving the linear system (7) $N_{I}$ times, one for each collocation node in $\Omega$, a sparse differentiation matrix $A$ is constructed which permits (1a) to be replaced by

$$
\underbrace{\left[\begin{array}{cc}
A_{I} & A_{B}
\end{array}\right]}_{A}\left[\begin{array}{c}
\boldsymbol{u}_{I} \\
\boldsymbol{u}_{B}
\end{array}\right]=-k_{c}^{2} \boldsymbol{u}_{I}
$$

where $\boldsymbol{u}_{I}=\left[u_{1}, \ldots, u_{N_{I}}\right]^{T}$ and $\boldsymbol{u}_{B}=\left[u_{N_{I+1}}, \ldots, u_{N}\right]^{T}$ are vectors containing the values of $u(\vec{r})$ at the collocation nodes in the domain $\Omega$ and on the boundary $\partial \Omega$, respectively.

Repeating the same procedure for the $N_{B}$ nodes on $\partial \Omega$, the Neumman $\mathrm{BC}$ is approximated by the homogeneous system

$$
\left[\begin{array}{ll}
B_{I} & B_{B}
\end{array}\right]\left[\begin{array}{c}
\boldsymbol{u}_{I} \\
\boldsymbol{u}_{B}
\end{array}\right]=\mathbf{0} .
$$

Incorporating (9) into (8) leads to the following ordinary eigenvalue problem for TE modes

$$
\left[A_{I}-A_{B}\left(B_{B}^{-1} B_{I}\right)\right] \boldsymbol{u}_{I}=-k_{c}^{2} \boldsymbol{u}_{I} .
$$

For the TM case, the Dirichlet BC can be directly imposed by simply letting $\boldsymbol{u}_{B}=0$ in (8).

The application of the RBF-FD method involves several parameters which should be carefully selected [5], [6]. The error of the method is strongly dictated by the highest degree of the appended polynomials, $d$. This is because the error associated to (6) is $O\left(h^{d-k+1}\right)$, where $h \propto(1 / \sqrt{N})$ is the internodal distance and $k$ is the order of the differential operator $L$. As mentioned above, for a given $d$, the total number of appended polynomials is $N_{p}=\left(\begin{array}{c}d+2 \\ d\end{array}\right)$. The stencil size is then taken as $N_{s} \gtrsim 2 N_{p}$. Lastly, the PHS exponent is commonly chosen as $m=2 d+1$.

\section{NUMERICAL RESULTS}

To illustrate the accuracy of the RBF-FD method, we consider the calculation of the cutoff wavenumbers of the first modes of an empty semicircular waveguide of radius $a$, shown in Fig. 2. The exact solutions of this problem can be found in $[8$, tables 1,2]. Fig. 3 depicts the relative error in the cutoff wavenumber for the first six $\mathrm{TM}_{p n}$ and six $\mathrm{TE}_{p n}$ modes versus the square root of the number of interior nodes, $\sqrt{N_{I}}$. The used RBF-FD parameters have been $d=5\left(N_{p}=21\right)$, $m=11$ and $N_{s}=45$. It can be seen that the $\mathrm{TM}_{p n}$ modes exhibit a fourth-order rate of convergence. In the TE case, the convergence rate reaches fifth-order accuracy. Figs. $4 \mathrm{a}$ and $4 \mathrm{~b}$ show the relative error in the cutoff wavenumber as a function of $\sqrt{N_{I}}$ for the the $\mathrm{TM}_{11}$ mode. In Fig $4 \mathrm{a}$ this error is plotted for several values of the PHS exponent, $m$. It can be seen that the rate of convergence does not change with $m$. In Fig. $4 b$ 

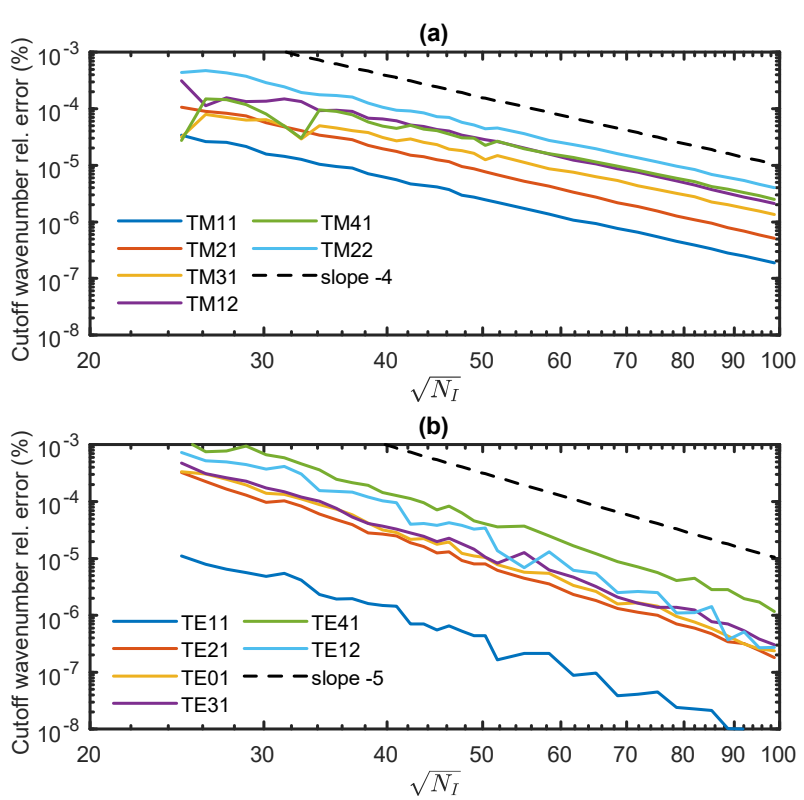

Fig. 3. Relative error in the cutoff wavenumber of TM (a) and TE (b) modes of a semicircular waveguide versus the square root of the number of interior nodes.
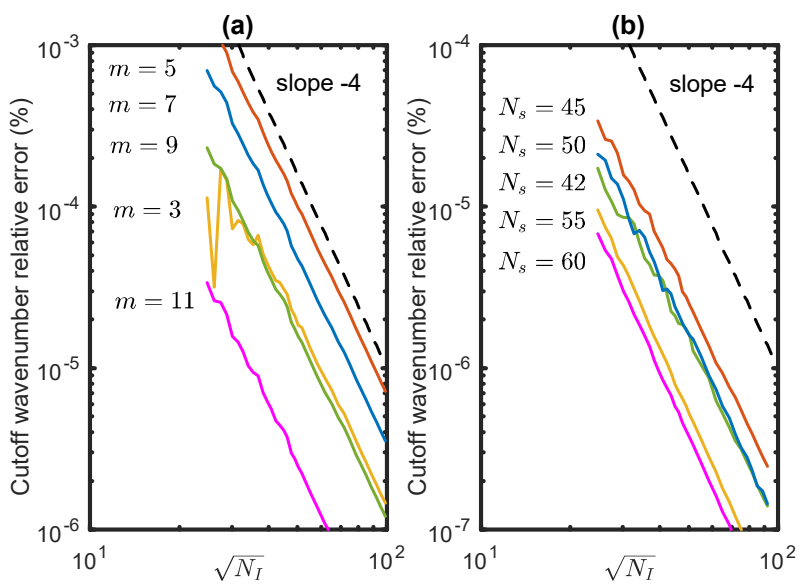

Fig. 4. Relative error in the cutoff wavenumber of the $\mathrm{TM}_{11}$ mode of a semicircular waveguide versus the square root of the number of interior nodes. (a) $d=5, N_{s}=45$. (b) $d=5, m=11$.

the error is plotted for several values of the stencil size $N_{s}$. Analogously to Fig. 4a, a vertical shifting in the error curves is only observed by varying $N_{s}$.

Secondly, we consider an eccentric circular waveguide with outer and inner conductors of radius $a$ and $b$, respectively. The offset between the centers of both conductors is $\ell$, as shown in Fig. 5a. The cross section of this waveguide is symmetric with respect to the $x$-axis. Consequently, even and odd modes can be calculated separately. For the sake of brevity, we will focus on the even TM modes. To this end, the upper half waveguide is solved with Dirichlet BCs on the conductors and Neumann BCs on the $x$-axis, as illustrated in Fig. $5 \mathrm{~b}$.

Fig. 6 depicts the longitudinal field, $E_{z}$, and the normalized cutoff wavenumber, $k_{c} a$, for the first six even TM modes of an eccentric circular waveguide with $b / a=0.5$ and $\ell / a=0.2$.

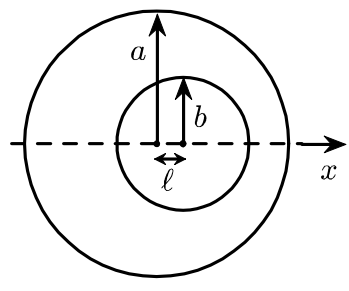

(a)

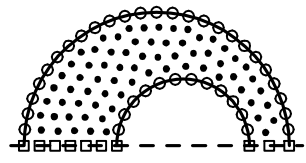

- Interior nodes

○ Dirichlet nodes

(b)
- Neumann nodes

Fig. 5. (a) Cross section of an eccentric circular waveguide. (b) Setup for the calculation of the even TM modes.
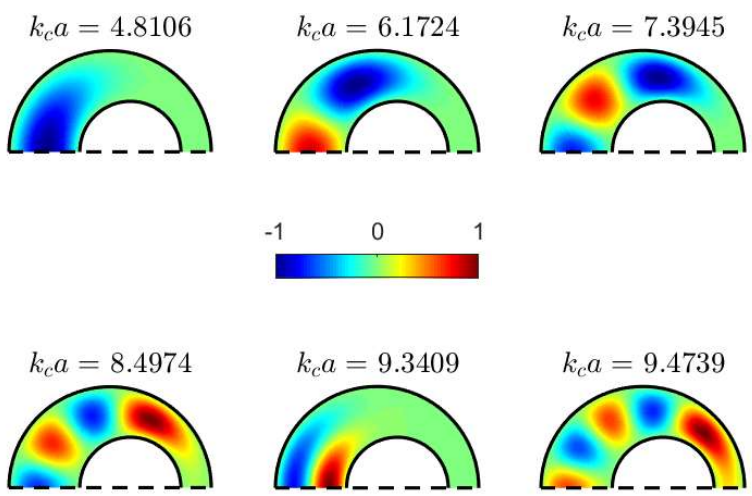

Fig. 6. Longitudinal field $E_{z}$ and normalized cutoff wavenumber $\left(k_{c} a\right)$ for the first six even TM modes of an eccentric circular waveguide with dimensions $b / a=0.5$ and $\ell / a=0.2$.

The RBF-FD parameters have been $d=5, m=11, N_{s}=50$ and $N_{I}=2448$. As shown in Table I, these results agree up to the digits shown with those given in [9, table III]. Moreover, the RBF-FD results outperform those calculated, also in [9], by the Finite Element Method (FEM) with 3028 degrees of freedom.

TABLE I. Normalized cutoff wavenumber $\left(k_{c} a\right)$ for the modes in Fig. 6.

\begin{tabular}{c|c|c}
\hline \hline This method & Method in [9] & FEM [9] \\
\hline \hline 4.8106 & 4.8106 & 4.8106 \\
6.1724 & 6.1724 & 6.1725 \\
7.3945 & 7.3945 & 7.3947 \\
8.4974 & 8.4974 & 8.4978 \\
9.3409 & 9.3409 & 9.3419 \\
9.4739 & 9.4739 & 9.4745 \\
\hline \hline
\end{tabular}

\section{CONCLUSION}

A RBF-FD method based on PHSs with supplementary polynomials has been applied to the analysis of homogeneous waveguides. It has been shown that this technique is able to provide high-order rates of convergence in problems with arbitrary shape domains and smooth solutions. Moreover, the RBF-FD method does not require the choice of an optimal shape parameter and its local nature makes it suitable to be applied to large-scale problems. The efficiency of the RBF-FD method can be improved by using it in subdomains with complex shapes while using classical FD stencils in the remaining part of the waveguide cross section. 


\section{REFERENCES}

[1] G. F. Fasshauer, Meshfree approximation methods with MATLAB, World Sci., River Edge, NJ, 2007.

[2] T. Kaufmann, Y. Yu, C. Engstrüm, Z. Chen, and C. Fumeaux, "Recent developments of the meshless radial point interpolation method for timedomain electromagnetics," Int. J. Numer. Model., vol. 25, no. 5-6, pp. 468-489, Sep.-Dec. 2012.

[3] S. Yang, Z. Chen, Y. Yu, and S. Ponomarenko, "A divergence-free meshless method based on the vector basis function for transient electromagnetic analysis", IEEE Trans. Microw. Theory Techn., vol.62, no. 7, pp.1409-1416, Jul. 2014.

[4] W. L. Nicomedes, K. J. Bathe, F. J. S. Moreira, and R. C. Mesquita, "Meshfree analysis of electromagnetic wave scattering from conducting targets: formulation and computations", Computers and Structures, vol.184, pp. 36-52, May 2017.

[5] N. Flyer, B. Fornberg, V. Bayona, and G.A. Barnett, "On the role of polynomials in RBF-FD approximations: I. Interpolation and accuracy," J. Comput. Phys., vol. 321, pp. 21-38, Sep. 2016.

[6] V. Bayona, N. Flyer, B. Fornberg, and G.A. Barnett, "On the role of polynomials in RBF-FD approximations: II. Numerical solution of elliptic PDEs," J. Comput. Phys., vol. 332, pp. 257-273, Mar. 2017.

[7] V. Lombardi, M. Bozzi, and L. Perregrini, "A novel variational meshless method with radial basis functions for waveguide eigenvalue problems," IEEE Trans. Microw. Theory Techn., vol. 66, no. 8, pp. 3714-3723, Aug. 2018.

[8] A. Elsherbeni, D. Kajfez, S. Zeng, "Circular sectoral waveguides," IEEE Antennas Propagat. Mag., vol. 33, no. 6, pp. 20-27, Dec. 1991.

[9] V. de la Rubia and J. Zapata, "An efficient method for determining TE and TM modes in closed waveguides made up of $\mathrm{N}$ cylindrical conductors," IEEE Trans. Microw. Theory Techn., vol. 53, no. 2, pp. 670-678, Feb. 2005. 